\title{
Frequency Salient Based Fiber Detection
}

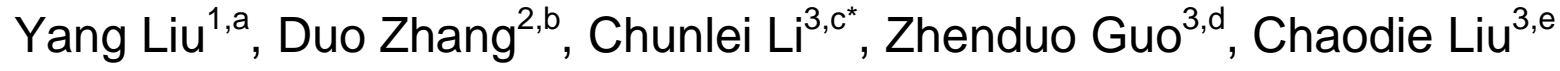 \\ ${ }^{1}$ School of Management and Economics, Beijing Institute of Technology, Beijing, 100081, China \\ ${ }^{2}$ School of Computer Science, Beijing Institute of Technology, Beijing 100081, China \\ ${ }^{3}$ School of Electric and Information Engineering, Zhongyuan Uniersity of Technology, Zhengzhou, Henan, \\ 450007, China \\ aemail: manutdryan11@163.com, bemail: zhangduo@sina.com, email: \\ lichunlei1979@sina.com, ${ }^{d e}$ ail: 33838402@qq.com, ${ }^{\mathrm{e} e m a i l: 1414500489 @ q q . c o m}$
}

Keywords: Fiber Anti-fake; Fiber Detection; Salient Image; Frequency tune

\begin{abstract}
The use of random and unique characteristics of the fiber distribution is an effective fiber anti-fake method in modern industry. Detecting and combing with the distribution of the fiber from images automatically can improve the efficiency of industrial production greatly. In this paper, we propose a frequency salient based fiber detection method which is robust to light condition and slightly background impurity. Our method exploits low level features of color and luminance, which is easy to implement, fast, and provides full resolution. The resulting saliency maps are better suited to fiber detection. The experiments are conducted on our released database, which include more than 10,000 fiber images, and the experimental results demonstrate the effectiveness of the proposed method.
\end{abstract}

\section{Introduction}

Anti-counterfeiting technology can be utilized to recognize the truth-false of products and guard against counterfeiting by extracting and recognizing features of products. The main anticounterfeiting technologies includes laser tag, digital label, texture anti-counterfeiting and wireless anti-counterfeiting, etc.

The texture anti-counterfeiting is widely used benefit from its property of difficult to replicate. Texture anti-counterfeiting mainly utilize the feature of randomness and the principle of uniqueness of patterns in nature such as fingerprint, wood grain, etc. People dispense fiber at the specified location of the paper pulp or the thin film by using the machine to create the anti-counterfeiting labels which have characteristics of random distribution and stability. It is almost impossible to dispense two identical anti-counterfeiting labels randomly in theory so that this way makes it hard to fake it. In the case of dispensing a large mount of fiber, anti-counterfeiting labels which have the characteristics of random distribution and stability will be generated because of unique texture features of the fiber and the characteristics of unpredictability and irregularities of the dispensed positions. In the industrial production line, anti-counterfeiting label image data which can meet the requirements will be automatically stored in the product database. Customers take photos of the product images and compare them with the images in the vendor database to verify the product's authenticity. The nature images are sensitive to illumination and are easily contaminated, this will greatly reduce performance of the retrieval based authentication method. In order to improve the precision of searching algorithm, the texture contrast scheme which has good Illumination robustness is needed. The framework of industrial fiber anti-counterfeiting is shown in Fig. 1.

Extracting and encoding the fiber texture information from images is an effective method for texture anti-counterfeiting. In the past decades, different methods are developed to extract object contour from images, such as edge detection and object sanlicy. Galun et.al [1] presented an algorithm for edge detection which utilizes efficient multiscale hierarchy of responses measuring the difference of oriented means of various lengths and orientations. They proposed an application to fiber detection and enhancement by utilizing stochastic completion-like process from both sides 
of fiber and identifies curved edges as concatenation of short straight response. Yang et.al [2] proposed an automated visual inspection system for the detection of foreign fibers in lint. The inspection algorithm contains four steps, namely, image enhancement, segmentation, post-processing and decision-making. Shi et.al[3] presented an automatic method for detecting fiber orientations of sewed carbon fibers in the production of fiber reinforced plastics. Detection was achieved by appropriate use of regional filling, edge detection operations, auto-correlation methods, and the Hough transformation. Kadir et.al [4] described a Gaussian mixture models and thresholding based method to remove foreign fibers and contaminants. Zhao et.al [5] proposed a novel edge detection algorithm based on multi-structure elements morphology of eight different directions. They got eight different edge direction results by using morphological gradient algorithm respectively, and final edge result was got by using synthetic weighted method.

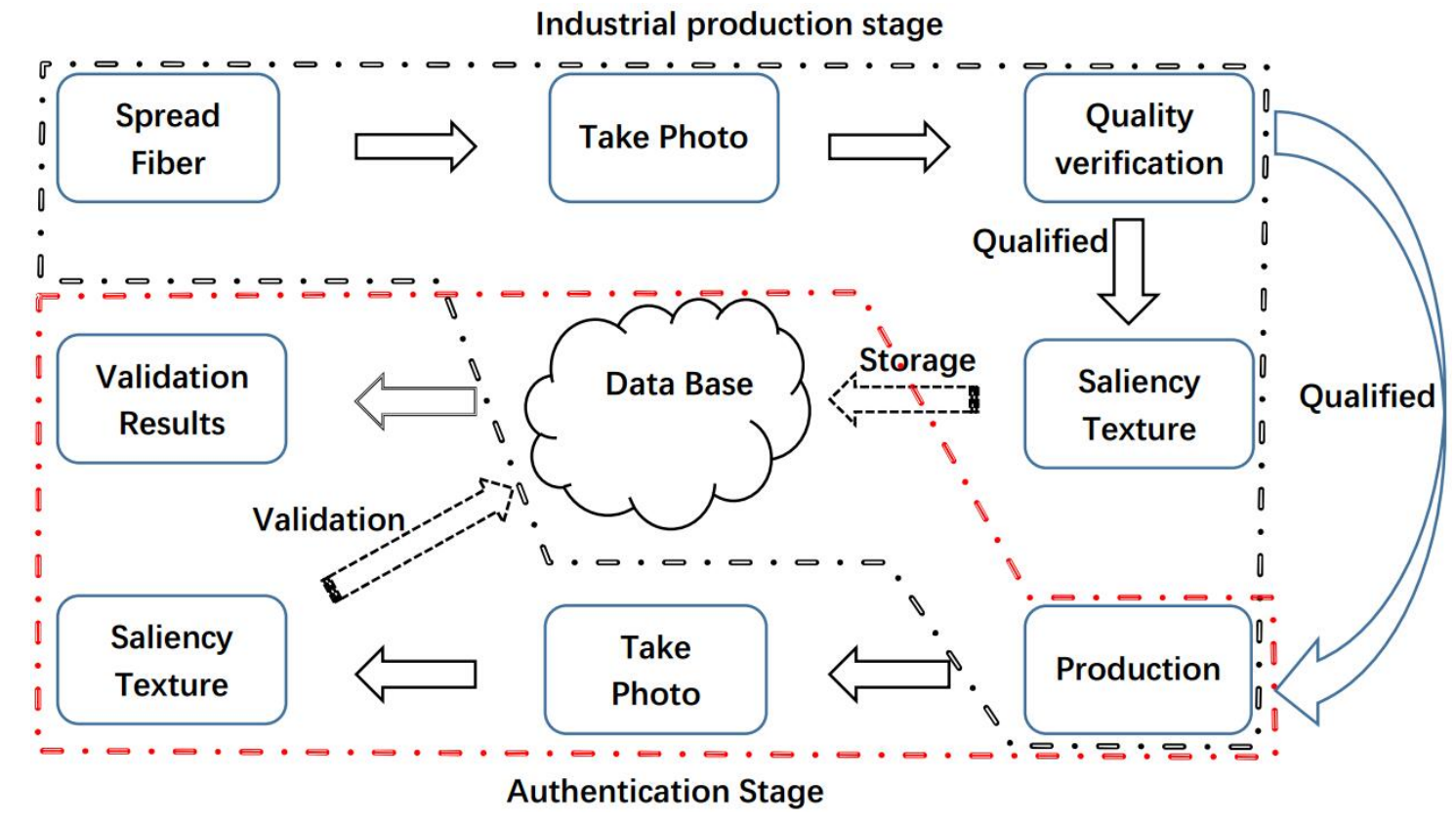

Fig. 1 Framework of industrial fiber anti-counterfeiting

Visual saliency measures to what extent a region attracts human attention. Recently, saliency are widely used in object detection. Xu et.al [6] proposed a novel method to detect salient regions from an image. This method uses joint embedding of spatial and color cues to detect pixel-level saliency, i.e., spatial constraint based saliency, color double-opponent saliency, and similarity distribution based saliency. Finally, a multi-layer structure is adopted to merge the three terms into a saliency map. Fu et.al [7] introduced an efficient and effective cluster-based co-saliency detection method. A global association constraint is enforced by clustering, avoiding the heavy learning. The final co-saliency maps are generated by fusing the single image saliency and multi-image saliency. Ren et.al [8] proposed a promising saliency detection approach, which can generate accurate saliency maps with well-defined object boundary. To avoid deficiency of pixel representation in large salient region detection and tolerate noise, they employ adaptive mean shift algorithm to extract perceptually and semantically meaningful super-pixels as features. Gao et.al [9] applied L0 smoothing to images to highlight fundamental image constituents while diminishing the insignificant details. Distance-transform based saliency detection is then performed on the smoothed image in order to extract the general salient regions, and the center-bias mechanism is used to form a rough saliency map. Finally, the rough saliency map is boundary-regulated to obtain the accurate object boundary by normalized-cut method. Chen et.al [10] proposed a region based saliency detection method following two successive steps, region weighting and contrast computing. With the application of region-level feature, region weights can be obtained by region weighting and adopted to contrast computing to achieve better results. 
In this paper, we present an effective frequency saliency based approach to detect the fibers automatically, our main contribution are as follows: (I) We present a method exploits low level features of color and luminance to detect the fivers, which is easy to implement, fast, and provides the full fiber contour. (2) We release an anti-counterfeiting database which includes more than 10,000 images captured by various mobile phone in different light condition.

\section{Fiber Saliency}

For a given image, let $w_{l c}$ be the low frequency cut-off value and $w_{\mathrm{hc}}$ be the high frequency cut-off value. To highlight large salient objects, we need to consider very low frequencies from the original image, i.e. $w_{l c}$ has to be low (first criterion). This also helps highlight salient objects uniformly. In order to have well defined boundaries, we need to retain high frequencies from the original images, i.e. $w_{\mathrm{hc}}$ has to be high. However, to avoid noise, coding artifacts, and texture patterns, the highest frequencies need to be disregarded. Since we are interested in a saliency map containing a wide range of frequencies, combining the outputs of several band pass filters with contiguous $\left[w_{l c}, w_{\mathrm{hc}}\right]$ pass bands is appropriate.

We choose the DoG filter for band pass filtering. The DoG filter is widely used in edge detection since it closely and efficiently approximates the LoG (Laplacian of Gaussian) filter, the DoG filter is given by Eq.1, where $\sigma_{1}$ and $\sigma_{2}$ are the standard deviations of the Gaussian $\left(\sigma_{1}>\sigma_{2}\right)$.

$$
\operatorname{DoG}(x, y)=\frac{1}{2 \pi}\left[\frac{1}{\sigma_{1}^{2}} e^{-\frac{\left(x^{2}+y^{2}\right)}{2 \sigma_{1}^{2}}}-\frac{1}{\sigma_{2}^{2}} e^{-\frac{\left(x^{2}+y^{2}\right)}{2 \sigma_{2}^{2}}}\right]
$$

If we define $\sigma_{1}=\rho \sigma$ and $\sigma_{2}=\sigma$ so that $\rho=\frac{\sigma_{1}}{\sigma_{2}}$, we find that a summation over DoG with standard deviations in the ratio $\rho$ results in:

$$
\sum_{n=0}^{N-1} G\left(x, y, \rho^{n+1} \sigma\right)-G\left(x, y, \rho^{n} \sigma\right)=G\left(x, y, \sigma \rho^{N}\right)-G(x, y, \sigma)
$$

For an integer $\mathrm{N} \geq 0$, which is simply the difference of two Gaussians whose standard deviations can have any ratio $\mathrm{K}=\rho^{\mathrm{N}}$. That is, we can obtain the combined result of applying several band pass filters by choosing a DoG with a large $\mathrm{K}$. If we assume that $\sigma_{1}$ and $\sigma_{2}$ are varied in such a way as to keep $\rho$ constant at 1.6, then we essentially add up the output of several edge detectors at several image scales. This gives an intuitive understanding of why the salient regions will be fully covered and not just highlighted on edges or in the center of the regions.

Based on the arguments in the previous section, a strategic selection of $\sigma_{1}$ and $\sigma_{2}$ will provide an appropriate band-pass filter to retain the desired spatial frequencies from the original image when computing the saliency map. With sufficiently long filters and a sufficiently large difference in $\sigma_{1}$ and $\sigma_{2}$, the passband of the resulting band-pass filter given in Eq.2 can be easily approximated from the two constituent Gaussians. With $\sigma_{1}>\sigma_{2}, w_{l c}$ is determined by $\sigma_{1}$ and $w_{\mathrm{hc}}$ is determined by $\sigma_{2}$. However, use of filters of a practical length, providing a correspondingly simple implementation, renders this approximation inaccurate.

The two $\sigma$ and therefore frequency parameters are therefore selected as follows. To implement a large ratio in standard deviations, we drive $\sigma_{1}$ to infinity. This results in a notch in frequency at DC while retaining all other frequencies. To remove high frequency noise and textures, we use a small Gaussian kernel keeping in mind the need for computational simplicity. For small kernels, the 
binomial filter approximates the Gaussian very well in the discrete case. We use $\frac{1}{16}$ giving $w_{\mathrm{hc}}=\pi / 2.75$.

Our method of finding the saliency map $\mathrm{S}$ for an image I of width $\mathrm{W}$ and height $\mathrm{H}$ pixels can thus be formulated as:

$$
S(x, y)=\left|I_{u}-I_{w_{h c}}(x, y)\right|
$$

Where $I_{u}$ is the arithmetic mean pixel value of the image and $I_{w_{h c}}$ is the Gaussian blurred version of the original image to eliminate fine texture details as well as noise and coding artifacts. The norm of the difference is used since we are interested only in the magnitude of the differences. This is computationally quite efficient. Also, as we operate on the original image without any down sampling, we obtain a full resolution saliency map.

To extend Eq.3 to use features of color and luminance, we rewrite it as:

$$
S(x, y)=\left\|\mathbf{I}_{\mu}-\mathbf{I}_{\mathrm{w}_{\mathrm{hc}}}(x, y)\right\|
$$

Where $\mathbf{I}_{\mu}$ is the mean image feature vector, $\mathbf{I}_{\mathrm{whc}_{\mathrm{hc}}}(x, y)$ is the corresponding image pixel vector value in the Gaussian blurred version of the original image, and $\|\bullet\|$ is the $L_{2}$ norm. Using the Lab color space, each pixel location is an $[L, a, b]^{T}$ vector, and the $L_{2}$ norm is the Euclidean distance.

\section{Experimental Results}

\section{Database Description}

Our database includes more than 10,000 images captured by several kinds cameras, such as iphone, mate7, note and mx5. The fiber image consists of different amount of long and short fiber. Due to the resolution of devices, the fiber image appear much in different, as shown in the first row of Fig. 2. We will release this database in the near future.

\section{Performance}

In order to remove the influence of the camera devices, guided image filtering algorithm [11] is adopted to enhance the image firstly. The performance of our proposed approach are as show in Fig. 2. From top to bottom, the first row are the original images, the second row shows the results after enhancement, and the third row presents the saliency fiber map.

From Fig. 2 (b), (c) and (d) we can see that the final saliency fiber map are insensitive to the camera devices and robust to the light variant in some extent. Our proposed algorithm can get the clearly fiber contour with the time cost less than one micro-seconds. 


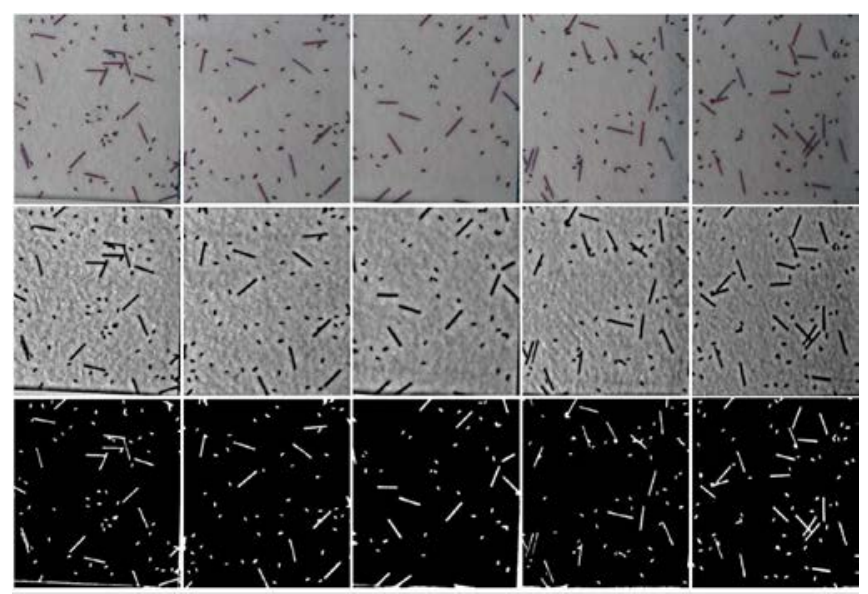

(a)

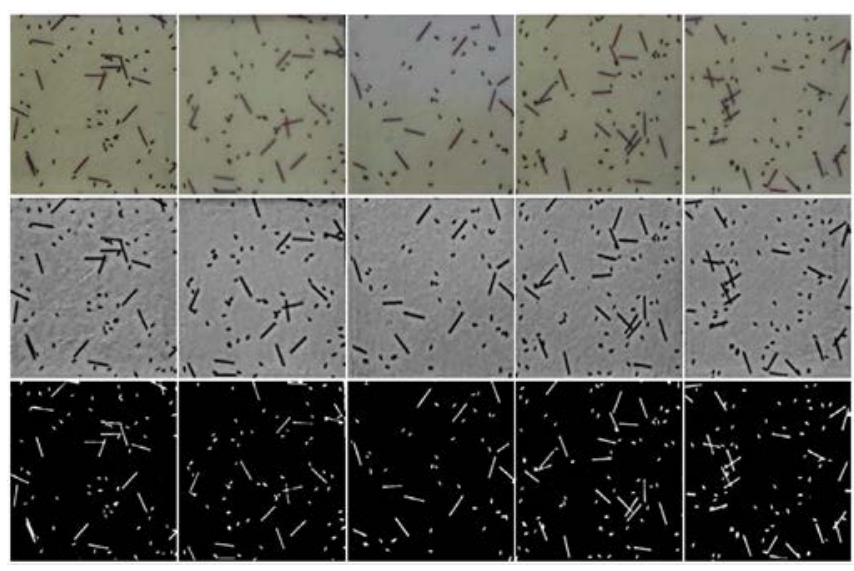

(c)

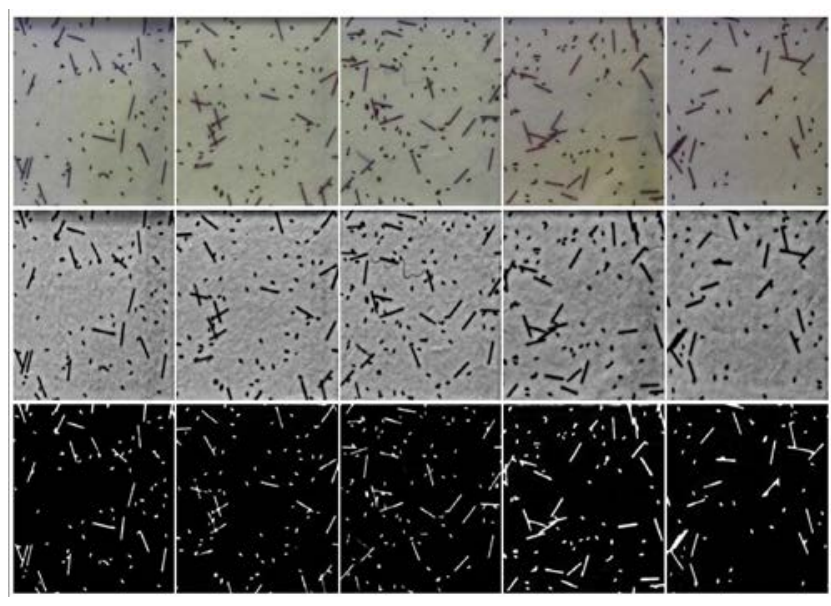

(b)

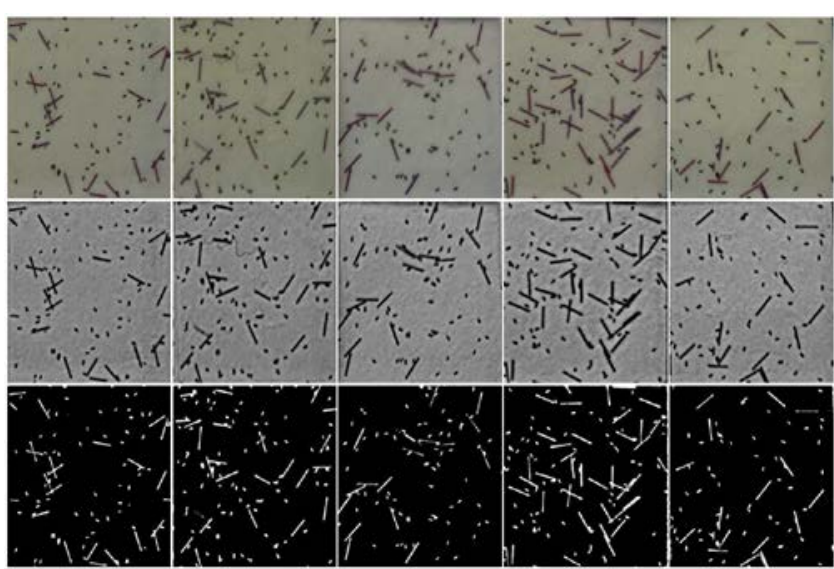

(d)

Fig. 2 Fiber Detection in different conditions, (a) captured by iphone, (b) captured by mate7, (c) captured by note, (d) captured by mx5. From top to bottom, the first row are the original images, the second row shows the image after enhancement, the third row shows the saliency fiber map.

\section{Conclusion}

In this paper, we present a frequency salient based fiber detection method to detect the fiber. The guide image filter is adopted to remove the influence of different devices, after that a method exploits low level features of color and luminance, which is easy to implement, fast, and provides full resolution. The experiments are conducted on our released database, and the experimental results demonstrate the effectiveness of the propose method.

\section{References}

[1] M.Galun, R.Basri, and A.Brandt, Multiscale edge detection and fiber enhancement using difference of oriented means[C], in International Conference on Computer Vision, 2007, pp. 1-8.

[2] W. Yang, D. Li, X. Wei, Y. Kang, and F. Li, An automated visual inspection sys-tem for foreign fiber detection in lint[J], in 2009 WRI Global Congress on Intelligent Systems, vol. 4, 2009, pp. 364-368. 
[3] L. Shi and S. Wu, Automatic fiber orientation detection for sewed carbon fibers, Tsinghua Science and Technology[J], vol. 12, no. 4, pp. 447-452, 2007.

[4] K. A. Peker and G.zsan, Contaminant and foreign fiber detection in cotton using gaussian mixture model[C], in International Conference on Application of Information and Communication Technologies, 2014, pp. 1-4.

[5] Y. Zhao, W. Gui, and Z. Chen, Edge detection based on multi-structure elements morphology[J], in World Congress on Intelligent Control and Automation, vol. 2, 2006, pp. 9795-9798.

[6] L. $\mathrm{Xu}, \mathrm{H} . \mathrm{Li}$, and Z. Wang, Saliency detection from joint embedding of spatial and color cues[C], in International Symposium on Circuits and Systems, 2012, pp. 2673-2676.

[7] H. Fu, X. Cao, and Z. Tu, Cluster-based co-saliency detection[J], IEEE Transactions on Image Processing, vol. 22, no. 10, pp. 3766-3778, 2013.

[8] Z. Ren, S. Gao, L. T. Chia, and I. W. H. Tsang, Region-based saliency detection and its application in object recognition[J], IEEE Transactions on Circuits and Systems for Video Technology, vol. 24, no. 5, pp. 769-779, 2014.

[9] H. Y. Gao and K. M. Lam, Segmentation-enhanced saliency detection model based on distance transform and center bias[C], in International Conference on Acoustics, Speech and Signal Processing, 2014, pp. 2803-2807.

[10] D. Chen, L. Ma, and R. Zhang, Region based saliency detection by learning back-ground information[C], in International Symposium on Broadband Multimedia Systems and Broadcasting, 2014, pp. 1-6.

[11] K. He, J. Sun, and X. Tang, Guided image filtering, in European Conference on Computer Vision: Part I, ser. ECCV'10, 2010. 